\title{
Quantificação de Ascósporos de Monosporascus cannonballus em Solos não cultivados de Caatinga e em áreas de cultivo de Melão do Rio Grande do Norte e Ceará
}

\author{
Erika V. Medeiros¹, Rui S. Júnior. ${ }^{1}$, Sami J. Michereff² \& Marcos R. Barbosa ${ }^{1}$ \\ ${ }^{1}$ Universidade Federal Rural do Semi-Árido, Cx. Postal 137, CEP 59625-900, Mossoró, RN, \\ e-mail: evmbio@hotmail.com; ${ }^{2}$ Universidade Federal Rural de Pernambuco, CEP 52171-900, Recife, PE
}

(Aceito para publicação em 04/10/2006)

Autor para correspondência: Érika Valente de Medeiros

\begin{abstract}
MEDEIROS, E.V., SALES JUNIOR, R., MICHEREFF, S.J. \& BARBOSA, M.R. Quantificação de ascósporos de Monosporascus cannonballus em solos não cultivados de Caatinga e em áreas de cultivo de melão do Rio Grande do Norte e Ceará. Fitopatologia Brasileira 31:500-504. 2006.
\end{abstract}

\section{RESUMO}

O fungo Monosporascus cannonballus é um importante patógeno radicular do meloeiro na região Nordeste, onde causa a doença denominada colapso. Como não existem informações sobre os níveis populacionais de ascósporos de $M$. cannonballus em solos brasileiros, realizou-se este trabalho com o objetivo de comparar as densidades de ascósporos em amostras solo de 15 áreas não cultivadas de Caatinga e 15 áreas produtoras de melão do Rio Grande do Norte e do Ceará. Em todos os solos analisados foram detectados ascósporos de $M$. cannonballus, sendo que as populações nas áreas não cultivadas variaram de 0,18 a 18,30 ascósporos. $\mathrm{g}^{-1}$ de solo e nas áreas cultivadas com meloeiro de 0,50 a 26,04 ascósporos. $\mathrm{g}^{-1}$ de solo. Não houve diferença significativa $(P=0,05)$ na densidade média de ascósporos entre solos não cultivados e cultivados. Entre as áreas cultivadas, a densidade média de ascósporos foi significativamente superior nas áreas com histórico de colapso causado por M. cannonballus, comparado às áreas sem histórico da doença. Pelos resultados alcançados há indícios que $M$. cannonballus não foi introduzido no Brasil por materiais de propagação, mas já era habitante natural dos solos de Caatinga antes da chegada da cultura do meloeiro.

Palavras-chave adicionais: Cucumis melo, colapso do meloeiro, extração de ascósporos, inóculo.

\begin{abstract}
Quantification of Monosporascus cannonballus ascospores in non-cultivated soils of Caatinga and melon producing fields in Rio Grande do Norte and Ceará States

The fungus Monosporascus cannonballus is an important melon root pathogen in the Northeast of Brazil, where it causes a disease called collapse. As information on population levels of $M$. cannonballus ascospores in Brazilian soils does not exist, this study aimed to compare ascospore density between soil samples of 15 non-cultivated areas of the Caatinga and 15 soil samples of melon producing areas in the States of Rio Grande do Norte and Ceará. Ascospores of M. cannonballus were detected in all soil samples analyzed. The density of the ascospores varied from 0.18 to 18.30 ascospores. $\mathrm{g}^{-1} \mathrm{of} \mathrm{soil}$ in non-cultivated Caatinga soil. Ascospore density in areas cultivated with melon varied from 0.50 to 26.04 ascospores. $\mathrm{g}^{-1}$ of soil. There was no significant statistical difference $(P=0.05)$ in the ascospore average density between cultivated and non-cultivated soil. Among the cultivated areas, the ascospore average density was significantly higher in the areas with a history of collapse caused by M. cannonballus when compared with areas without a disease history. There is evidence that M. cannonballus was not introduced in Brazil by propagation material, but is possibly a natural inhabitant of non-cultivated soils of Caatinga.

Additional keywords: Cucumis melo L., melon collapse, ascospore extraction, inoculum.
\end{abstract}

O fungo Monosporascus cannonballus Pollack \& Uecker (Ascomycota, Sordariomycetidae) é um importante patógeno radicular do meloeiro (Cucumis melo L.) na região Nordeste, o principal pólo produtor brasileiro (Andrade et al., 2005). Isoladamente ou em associação com outros

\footnotetext{
* Parte da Dissertação de Mestrado da primeira autora. Universidade Federal Rural do Semi-Árido. Mossoró RN. 2005.
}

patógenos causa a doença denominada colapso ou declínio, cujos sintomas incluem amarelecimento e morte das folhas mais velhas, com gradual declínio das ramas, seguido de murcha e morte das plantas na época próxima à formação dos frutos, como conseqüência da necrose do sistema radicular e perda de muitas raízes secundárias e terciárias (Martyn \& Miller, 1996).

De fase assexuada ainda desconhecida, $M$. cannonballus é um ascomiceto habitante do solo, que 
apresenta peritécios globosos, pretos, com ascos clavados a piriformes, contendo apenas um ascósporo por asco. Os ascósporos são asseptados, globosos, pretos, lisos, com parede espessa, medem de 30 a $50 \mu \mathrm{m}$ de diâmetro (Sivanesan, 1991) e têm capacidade de sobreviver no solo por longos períodos na ausência de hospedeiro, constituindo o inóculo primário para as infecções radiculares (Martyn \& Miller, 1996; Stanghellini et al., 1996; Waugh et al., 2003).

O monitoramento do inóculo de $M$. cannonballus no solo é uma medida que pode auxiliar no manejo do colapso do meloeiro (Mertely et al., 1993; Waugh et al., 2003). Como inexistem meios seletivos para isolamento desse organismo, o método da extração física pela flotação de sacarose tem sido aplicado com várias modificações para detecção e quantificação dos ascósporos no solo (Stanghellini \& Rasmussen, 1992; Mertely et al., 1993; Beltrán et al., 2005).

No Brasil, M. cannonballus foi detectado pela primeira vez em 2002, em áreas de cultivo de melão nos estados do Rio Grande do Norte (RN) e Ceará (CE) (Sales Jr. et al., 2003). Em levantamentos conduzidos nessa região no ano seguinte, o fungo foi isolado de $30 \%$ das áreas apresentando colapso (Andrade et al., 2005), evidenciando a magnitude do problema e a necessidade da adoção de medidas integradas de manejo da doença. Como não existem informações sobre os níveis populacionais de ascósporos de M. cannonballus em solos brasileiros, objetivou-se nesse trabalho quantificar e comparar as densidades de ascósporos em solos não cultivados de Caatinga e em solos de áreas produtoras de melão do $\mathrm{RN}$ e $\mathrm{CE}$.

Amostras de solo foram coletadas em 15 áreas não cultivadas de Caatinga e 15 áreas produtoras de melão, localizadas na região semi-árida dos estados do $\mathrm{RN}$ e $\mathrm{CE}$, no período de março a abril de 2005 (Tabela 1). Cada amostra foi composta por cinco sub-amostras, coletadas em ziguezague, à profundidade de $10 \mathrm{a} 20 \mathrm{~cm}$, onde se encontra a maior concentração de ascósporos de M. cannonballus (Mertely et al., 1993). Nas áreas produtoras de melão, foram obtidas informações sobre o histórico da ocorrência de colapso causado por M. cannonballus.

As amostras de solo foram secas em ambiente coberto e depois peneiradas em malha de $2 \mathrm{~mm}$ para retirada de resíduos. De cada amostra de solo foram retiradas seis sub-amostras, constituindo as repetições, das quais os ascósporos de M. cannonballus foram extraídos pelo método de flotação de sacarose, conforme Beltrán et al. (2005). Inicialmente, as amostras foram peneiradas em uma malha de $250 \mu \mathrm{m}$, eliminando-se as partículas retidas. Uma amostra de $20 \mathrm{~g}$ de solo peneirado foi colocada em $500 \mathrm{ml}$ de água, agitada durante 5 min e depois passada por peneiras com malhas de 75 e $30 \mu \mathrm{m}$. As partículas retidas na malha de $30 \mu$ mforam lavadas em água corrente e centrifugadas a $900 \mathrm{~g}$, durante $4 \mathrm{~min}$. O sobrenadante foi descartado e as partículas foram dissolvidas em $40 \mathrm{ml}$ de solução de sacarose a $50 \%$ e centrifugadas a $900 \mathrm{~g}$, durante $2 \mathrm{~min}$. As partículas restantes foram dissolvidas em solução de sacarose a $50 \%$ e centrifugadas. O sobrenadante foi passado por uma malha de $30 \mu \mathrm{m}$ e as partículas retidas distribuídas em placas de Petri, procedendo-se à contagem dos ascósporos característicos em um microscópio estereoscópico a 60x.

Os dados obtidos foram transformados em $\sqrt{x+1}$ e submetidos à análise de variância. As médias dentro das áreas de Caatinga e dentro das áreas cultivadas foram comparadas pelo teste de Scott-Knott $(P=0,05)$, enquanto o teste $t$ para amostras independentes $(P=0,05)$ foi utilizado na comparação das médias entre áreas de Caatinga e cultivadas, bem como entre áreas com e sem histórico da doença dentro das cultivadas.

Ascósporos de $M$. cannonballus foram detectados em todos os solos analisados do RN e do CE, incluindo áreas não cultivadas de Caatinga e cultivadas com meloeiro (Tabela 1), confirmando a observação de que esse fungo é habitante de solos de regiões quentes, áridas e semi-áridas (Martyn \& Miller, 1996; Cohen et al., 2000; Pivonia et al., 2002; Stanghellini et al., 2004).

As populações em áreas não cultivadas variaram de 0,18 a 18,30 ascósporos. $\mathrm{g}^{-1}$ de solo, sendo que em $60 \%$ das áreas o nível populacional foi superior a 3 ascósporos. $\mathrm{g}^{-1}$ rama de solo. Em áreas cultivadas com meloeiro, as

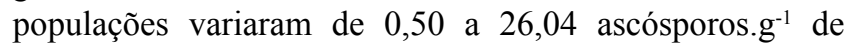
solo e $40 \%$ das áreas apresentaram níveis superiores a 3 ascósporos. $\mathrm{g}^{-1}$ de solo (Tabela 1). Esses níveis populacionais são muitos elevados quando comparados aos registrados em outros estudos em que a mesma metodologia de extração foi utilizada. No Arizona (EUA), 76\% das áreas cultivadas com meloeiro e com histórico da doença apresentaram até 3 ascósporos. $\mathrm{g}^{-1}$ de solo e somente $3 \%$ das áreas apresentaram 6 ascósporos. $\mathrm{g}^{-1}$ de solo, o máximo registrado. Além disso, a média de duas áreas de deserto sem cultivo foi de 1,08 ascósporos. $\mathrm{g}^{-1}$ de solo (Stanghellini et al., 1996). O nível populacional máximo de $M$. cannonballus no Texas (EUA) foi de 14,40 ascósporos.g ${ }^{-1}$ de solo (Mertely et al., 1993), na Califórnia (EUA) foi de 2,10 ascósporos. $\mathrm{g}^{-1}$ de solo (Radewald et al., 2004) e na Espanha foi de 2,34 ascósporos. $\mathrm{g}^{-1}$ de solo (Beltrán et al., 2005), todos em áreas cultivadas com meloeiro.

Houve diferença significativa $(P=0,05)$ nos níveis populacionais de ascósporos de $M$. cannonballus entre as áreas não cultivadas (Caatinga), bem como entre as áreas cultivadas com meloeiro. Por outro lado, não foi constatada diferença significativa na densidade média de ascósporos entre solos não cultivados (4,27 ascósporos. $\mathrm{g}^{-1} \mathrm{de}$ solo) e cultivados (5,25 ascósporos. $\mathrm{g}^{-1}$ de solo), assemelhando ao registrado no deserto do Arizona (Stanghellini et al., 1996).

Entre as áreas cultivadas com meloeiro, a densidade média de ascósporos foi significativamente superior nas áreas com histórico de colapso causado por $M$. cannonballus (7,94 ascósporos. $\mathrm{g}^{-1}$ de solo) comparado às áreas sem histórico da doença (2,17 ascósporos. $\mathrm{g}^{-1}$ de solo), divergindo do verificado no Arizona, onde populações similares foram observadas em campos com e sem histórico de colapso (Stanghellini et al., 1996). 
TABELA 1 - População de ascósporos de Monosporascus cannonballus em amostras de solo provenientes de ecossistema de Caatinga e áreas de produção de meloeiro dos estados do Rio Grande do Norte e Ceará

\begin{tabular}{|c|c|c|c|c|}
\hline Ambiente & Código & Local & $\begin{array}{l}\text { Histórico de } \\
\text { colapso }{ }^{1}\end{array}$ & $\begin{array}{c}\text { População ( ascósporos.g } \\
\text { solo) }^{2}\end{array}$ \\
\hline \multirow{16}{*}{$\begin{array}{c}\text { Solo de Caatinga não } \\
\text { cultivado }\end{array}$} & - $\mathrm{C}-01$ & Mossoró, RN & - & $18,30 \mathrm{a}$ \\
\hline & C-02 & $\mathrm{Km} 0, \mathrm{RN}$ & - & $7,00 \mathrm{~b}$ \\
\hline & C-03 & Baraúnas, RN & - & $6,82 \mathrm{~b}$ \\
\hline & C-04 & Tabuleiro das Russas, CE & - & $5,92 \mathrm{c}$ \\
\hline & C-05 & Tabuleiro das Russas, CE & - & $5,04 \mathrm{c}$ \\
\hline & C-06 & Assú, RN & - & $4,34 \mathrm{~d}$ \\
\hline & C-07 & Mossoró, RN & - & $3,67 \mathrm{~d}$ \\
\hline & C-08 & Lagoa do Piató, RN & - & $3,12 \mathrm{~d}$ \\
\hline & C-09 & Baraúnas, RN & - & $3,10 \mathrm{~d}$ \\
\hline & C-10 & Lagoa Virgem, RN & - & $2,58 \mathrm{e}$ \\
\hline & C-11 & Assú, RN & - & $1,81 \mathrm{e}$ \\
\hline & $\mathrm{C}-12$ & Macau, RN & - & $1,60 \mathrm{e}$ \\
\hline & $\mathrm{C}-13$ & Pendências, RN & - & $0,31 \mathrm{f}$ \\
\hline & C-14 & Macau, RN & - & $0,22 \mathrm{f}$ \\
\hline & $\mathrm{C}-15$ & Alto do Rodrigues, RN & - & $0,18 \mathrm{f}$ \\
\hline & Média & - & & $4,27 \mathrm{~A}$ \\
\hline \multirow{16}{*}{$\begin{array}{l}\text { Solo cultivado } \\
\text { com meloeiro }\end{array}$} & M-01 & $\mathrm{Km} \mathrm{0,} \mathrm{RN}$ & + & $26,04 \mathrm{a}$ \\
\hline & M-02 & Baraúnas, RN & + & $11,98 \mathrm{~b}$ \\
\hline & M-03 & Baraúnas, RN & + & $7,58 \mathrm{c}$ \\
\hline & M-04 & $\mathrm{Km} 0, \mathrm{RN}$ & + & $6,06 \mathrm{~d}$ \\
\hline & M-05 & $\begin{array}{l}\text { Tabuleiro das Russas, } \\
\text { CE }\end{array}$ & - & $5,77 \mathrm{~d}$ \\
\hline & M-06 & $\begin{array}{l}\text { Tabuleiro das Russas, } \\
\text { CE }\end{array}$ & + & $5,50 \mathrm{~d}$ \\
\hline & M-07 & Mossoró, RN & - & $2,73 \mathrm{e}$ \\
\hline & M-08 & Assú, RN & - & $2,61 \mathrm{e}$ \\
\hline & M-09 & Mossoró, RN & + & $2,54 \mathrm{e}$ \\
\hline & M-10 & Macau, RN & + & $1,98 \mathrm{e}$ \\
\hline & M-11 & Quixeré, CE & + & $1,87 \mathrm{e}$ \\
\hline & M-12 & Mossoró, RN & - & $1,78 \mathrm{e}$ \\
\hline & M-13 & Assú, RN & - & $1,19 \mathrm{f}$ \\
\hline & M-14 & Assú, RN & - & $0,64 \mathrm{f}$ \\
\hline & M-15 & Baraúnas, RN & - & $0,50 \mathrm{f}$ \\
\hline & Média & - & & $5,25 \mathrm{~A}$ \\
\hline
\end{tabular}

Um rápido aumento na densidade do inóculo de $M$. cannonballus no solo tem sido associado com a morte das raízes do meloeiro, a partir de duas semanas da colheita e atingindo o máximo com dois a quatro meses após a morte das plantas, o que pode ser conseqüência da redução nos níveis de nutrientes disponíveis e estímulo à formação de estruturas reprodutivas (Waugh et al., 2003; Stanghellini et al., 2004; Beltrán et al., 2005). Outros fatores, como temperatura, umidade do solo, e manejo da cultura, também exercem grande influência sobre a população de $M$. cannonballus no solo. Elevadas densidades de ascósporos têm sido registradas em solos com temperaturas entre 25 e 30 
${ }^{\circ} \mathrm{C}$ (Pivonia et al., 2002; Waugh et al., 2003) e sem saturação de umidade (Beltrán et al., 2005). Em estudo realizado no Texas, o manejo intensivo da cultura do meloeiro, como cultivos sucessivos sem rotação, uso de irrigação por gotejamento e de cobertura plástica ("mulch"), propiciou um rápido aumento da densidade de inóculo de M. cannonballus quando comparado ao manejo tradicional, caracterizado pelo uso da rotação de culturas com milho e cebola, irrigação por sulco e solo descoberto(Mertely et al., 1993).

Como o colapso do meloeiro é considerado uma doença monocíclica devido ao patógeno não possuir uma fase anamórfica conhecida e formar ascósporos principalmente no final do ciclo da cultura, a densidade inicial de inóculo de M. cannonballus no solo poderia ser relevante para prever os riscos futuros de doença no campo (Beltrán et al., 2005). Ao avaliarem o potencial reprodutivo de $M$. cannonballus no solo, Waugh et al. (2003) concluíram que campos eram considerados problemáticos quando apresentavam no mínimo 2 ascósporos. $\mathrm{g}^{-1}$ de solo. Caso esse limiar de risco fosse considerado nas áreas avaliadas no RN e CE, 67\% das áreas não cultivadas e $60 \%$ das cultivadas teriam risco de serem problemáticas quanto à incidência de colapso. No entanto, 20\% das áreas cultivadas (M-05, M-07 e M08 ) com níveis populacionais superiores ao limite de risco ( 2 ascósporos. g $^{-1}$ de solo) não apresentaram histórico de ocorrência do colapso causado por M. cannonballus (Tabela 1), o que reforça a abordagem de Mertely et al. (1993), na qual a densidade de inóculo é apenas um dos componentes do potencial de inóculo desse microrganismo, que envolve também a viabilidade e a infectividade dos ascósporos.

Embora os ascósporos de M. cannonballus possam ser extraídos do solo, não germinam ou raramente germinam em condições de laboratório, o que dificulta determinar quantitativamente a viabilidade de ascósporos de populações residentes no solo (Stanghellini et al., 1996). A infectividade dos ascósporos é difícil de ser avaliada em populações residentes, pois cada propágulo possui sua infectividade inerente, que é influenciada por vários aspectos do solo, como condições de temperatura e umidade, fontes e níveis de nutrientes disponíveis, e atividade microbiana (Stanghellini et al., 1996; Pivonia et al., 2002; Waugh et al., 2003; Beltrán et al., 2005). Além disso, a planta hospedeira também exerce influência sobre a infectividade dos ascósporos de M. cannonballus, pela qualidade e quantidade de exsudatos radiculares estimulantes liberados, bem como pelo nível de predisposição à infecção devido ao estresse hídrico (Pivonia et al., 1997). Portanto, existem muitas influências sobre o potencial de inóculo que podem resultar na ausência de correlação entre densidade populacional de $M$. cannonballus no solo e intensidade do declínio no cultivo (Stanghellini et al., 2004).

Com os resultados alcançados nesse estudo foi demonstrado que $M$. cannonballus não foi introduzido no Brasil por materiais de propagação, mas já era habitante natural dos solos semi-áridos de Caatinga antes da chegada da cultura do meloeiro. As densidades populacionais verificadas nas áreas não cultivadas constituem um alerta para o potencial destrutivo que pode representar o colapso do meloeiro com a expansão ou abertura de novas áreas de plantio, principalmente considerando que a migração para áreas novas de Caatinga é uma prática comum dos produtores quando a produtividade é comprometida devido a doenças radiculares. Outro aspecto importante a considerar é que, a densidade de ascósporos de M. cannonballus detectada no solo é um indicativo do risco, mas não o fator determinante para a ocorrência de epidemia de colapso, pois a viabilidade e a infectividade desses propágulos é influenciada por vários fatores. Como o inóculo de $M$. cannonballus já existia quando a cultura do meloeiro foi introduzida em áreas de Caatinga desmatadas e os níveis de incidência do colapso nos cultivos se mantiveram em níveis pouco alarmantes até 2002, o aumento significativo da doença pode estar associado à mudança na tecnologia de produção. Nos últimos anos, nas áreas de produção de melão do RN e CE têm sido adotadas tecnologias que preconizam a exploração intensiva, tais como monocultura, irrigação por gotejamento, aumento da densidade de plantio e uso de cobertura plástica ("mulch"), que comprovadamente propiciam as condições favoráveis para o aumento da infectividade dos ascósporos de $M$. cannonballus e do desenvolvimento do colapso (Bruton, 1998). Portanto, há necessidade de estudos sobre fatores que não permitam ao inóculo do fungo presente nas áreas expressar sua máxima potencialidade, representada pela infecção do hospedeiro e desenvolvimento de epidemias do colapso.

\section{REFERÊNCIAS BIBLIOGRÁFICAS}

ANDRADE, D.E.G.T., MICHEREFF, S.J., BIONDI, C.M., NASCIMENTO, C.W.A. \& SALES JR., R. Freqüência de fungos associados ao colapso do meloeiro e relação com características físicas, químicas e microbiológicas dos solos. Summa Phytopathologica 31:326-331. 2005.

BELTRÁN, R., VINCENT, A., SALES JR., R., GARCÍAJIMÉNEZ, J. \& ARMENGOL, J. Population dynamics of Monosporascus cannonballus ascospores in marsh soils in eastern Spain. European Journal of Plant Pathology 113:357-365. 2005.

BRUTON, B.D. Soilborne diseases in cucurbitaceae: pathogen virulence and host resistance. In: McCreight, J. (Ed.) Cucurbitaceae '98. Alexandria. International Society of Horticultural Science. 1998. pp.143-166.

COHEN, R., PIVONIA, S., BURGER, Y., EDELSTEIN, M., GAMLIEL, A. \& KATAN, J. Toward integrated management of Monosporascus wilt of melons in Israel. Plant Disease 84:496-505. 2000.

MARTYN, R.D. \& MILLER, M.E. Monosporascus root rot and vine decline: an emerging disease of melon worldwide. Plant Disease 80:716-725. 1996.

MERTELY, J.C., MARTYN, R.D., MILLER, M.E. \& BRUTON, B.D. Quantification of Monosporascus cannonballus ascospores in three commercial muskmelon fields in south Texas. Plant Disease 77:766-771. 1993. 
PIVONIA, S., COHEN, R., KAFKAFI, U., BEM-ZE'EV, I.S. \& KATAN, J. Sudden wilt of melons in southern Israel: fungal agents and relationship with plant development. Plant Disease 81:12641268. 1997.

PIVONIA, S., COHEN, R., RIGEL, J. \& KATAN, J. Effect of soil temperature on disease development in melon plants infected by Monosporascus cannonballus. Plant Pathology 51:472-479. 2002.

RADEWALD, K.C., FERRIN, D.M. \& STANGHELLINI, M.E. Sanitation practices that inhibit reproduction of Monosporascus cannonballus in melon roots left in the field after crop termination. Plant Pathology 53:660-668. 2004.

SALES JR., R., OLIVEIRA, O.F., SENHOR, R.F. \& ALVES, M.Z. Monosporascus cannonballus agente causal do colapso em plantas de melão no Rio Grande do Norte, Brasil. Fitopatologia Brasileira 28:567. 2003.

SIVANESAN A. Monosporascus cannonballus. Mycopathologia
114:53-54. 1991.

STANGHELLINI, M.E. \& RASMUSSEN, S.L. A quantitative method for the recovery of ascospores of Monosporascus cannonballus from field soil. Phytopathology 82:1115. 1992.

STANGHELLINI, M.E., KIM, D.H. \& RASMUSSEN, S.L. Ascospores of Monosporascus cannonballus: germination and distribution in cultivated and desert soils in Arizona. Phytopathology 86:509-514. 1996.

STANGHELLINI, M.E., WAUGH, M.M., RADEWALD, K.C., KIM, D.H., FERRIN, D.M. \& TURINI, T. Crop residues destruction strategies that enhance rather than inhibit reproduction of Monosporascus cannonballus. Plant Pathology 53:50-53. 2004.

WAUGH, M.M., KIM, D.H., FERRIN, D.M. \& STANGHELLINI, M.E. Reproductive potential of Monosporascus cannonballus. Plant Disease 87:45-50. 2003. 